\title{
Monitoring EDTA and endogenous metabolite biomarkers from serum with mass spectrometry
}

\author{
Rachel Lowe ${ }^{\mathrm{a}, \mathrm{b}}$, Eden P. Go ${ }^{\mathrm{a}}$, Grace C. Tong ${ }^{\mathrm{a}}$, Nicolas H. Voelcker ${ }^{\mathrm{b}}$ and Gary Siuzdak $^{\mathrm{a}, *}$ \\ ${ }^{a}$ Department of Molecular Biology, The Scripps Center of Mass Spectrometry, The Scripps Research \\ Institute, 10550 North Torrey Pines Road, La Jolla, CA 92037, USA \\ ${ }^{\mathrm{b}}$ School of Chemistry Physics and Earth Sciences, Flinders University, Bedford Park, \\ South Australia, 5042
}

\begin{abstract}
We describe a quantitative method for the determination of ethylenediaminetetraacetic acid (EDTA) in human serum by gas chromatography mass spectrometry (GC-MS), liquid chromatography electrospray ionisation tandem mass spectrometry (LC-ESI-MS/MS), and desorption ionisation on silicon mass spectrometry (DIOS-MS). In the initial stages of the analysis, endogenous metabolites (1-palmitoyl-sn-glycero-3-phosphocholine and 1-stearoyl-sn-glycero-3-phosphocholine) were readily observed in LC-ESI-MS and DIOS-MS however, direct analysis of the EDTA free acid had limited sensitivity. In order to improve EDTA detection we employed a straightforward esterification derivatization. The most successful derivatization procedure converted EDTA to its methyl ester and, since ${ }^{13} \mathrm{C}$ isotopes of these reagents are readily available, internal standards could be easily generated for quantitative analysis. This approach provided a limit of detection of 0.5 and $0.1 \mu \mathrm{M}$ for GC-MS and LC-ESI-MS, and offers a viable method for the EDTA detection.
\end{abstract}

\section{Introduction}

Ethylenediaminetetraacetic acid (EDTA) is a metal chelating agent that has been used extensively for blood preservation [7]. When mixed with blood, EDTA immediately chelates the available calcium preventing the formation of fibrin thereby inhibiting coagulation [7]. The concentration of EDTA in preserved blood tubes varies according to volume but usually is in the concentration range of 3-7 $\mathrm{mM}$ [6]. This high concentration of EDTA is an advantage in its analyses in criminal cases when the origin of blood evidence is under dispute.

However, there are many factors that inhibit the analysis of EDTA including its ubiquitous use in a variety of industries. Since its commercialization in the 1950's [17], EDTA has been widely utilized as a food colouring preservative [3], a water softener in laundry detergents [3] and a metal chelating agent in photography [14]. This has resulted in the need of both sensitive and accurate methods of analysis and the careful use of controls.

While there have been numerous advances in the analysis of EDTA, focus of research has been placed on EDTA identification in its free acid state. EDTA detection in its free state is inhibited by its distribution among chromatographic peaks that correspond to several different metal species to which it may

\footnotetext{
*Corresponding author. E-mail: siuzdak@scripps.edu.
} 
chelate. One reasonable approach is to convert all available EDTA to a single compound is to add excess of one metal that strongly complexes with EDTA. The effectiveness of this method is highly dependent on $\mathrm{pH}$ and the type of metal ion used, typically iron (III) [2], copper (II) [4,13] and nickel [12]. Moreover, due to its non-volatile nature and polarity, typical methods of analysis such as liquid chromatography mass spectrometry have been used for EDTA detection [10]. One of the most significant advances in EDTA detection was made using capillary electrophoresis mass spectrometry (CE/MS), Sheppard and Henion achieved detection levels of $0.025 \mu \mathrm{M}$ [10]. It should be noted however, that these authors used an in-house adapted liquid junction interface not commonly available.

Another avenue of investigation in an attempt to optimise detection levels is utilising gas chromatography (GC). In order for this method to be viable for EDTA analysis, the molecule must be relatively volatile and thermally stable. To accomplish this, the carboxylic acid groups in EDTA can be converted to its ester via its reaction with an acidified alcohol. Esterification has been reported earlier for military applications [1] and water analysis [14], however, the detection levels reported for these methods were not ideal for dried bloodstain analysis in forensics.

Based on the current approaches used for EDTA analysis, it is apparent that there is a need for the development of an easily accessible method of detection that will enable more straightforward analysis of EDTA. In this study, we describe a simple derivatization procedure that enhances EDTA detection and assess its applicability to three mass spectrometry platforms including GC-MS, LC-ESI-MS/MS and DIOS-MS. During the course of these studies, two ubiquitous endogenous metabolites were observed which could act as biomarkers for blood as an alternative to DNA testing [5].

\section{Experimental methods}

\subsection{Materials}

Optima grade methanol, optima grade acetonitrile, optima grade hydrochloric acid, and $49 \%$ hydrofluoric acid were obtained from Fisher Scientific. $\left[{ }^{13} \mathrm{C}\right]$-Methanol with purity of $>95 \%$, 1-butanol of 99.9\%, formic acid of 95\%, Biotech grade 99.9\% dichloromethane and EDTA (MW 292.24) of $99.995 \%$ purity and were purchased from Sigma Aldrich. EDTA was dissolved in nanopure water ( $\mathrm{pH} \mathrm{8}$, adjusted with ammonium hydroxide (Fisher Scientific)) to obtain the stock solution $(1000 \mu \mathrm{M})$. Serum (male $\mathrm{AB}$ human serum, sterile filtered) used in the quantitative analysis was obtained from Sigma. Absolute ethanol was purchased from AAPER alcohol and Chemical Company and pentafluorophenylpropyldimethylchlorosilane used for surface modification was purchased from Gelest, Inc.

\subsection{EDTA extraction from complex mixtures}

Cold methanol $(100 \mu \mathrm{l})$ was added to EDTA spiked serum samples $(50 \mu \mathrm{l})$ and the mixture was mixed by vortexing for $1 \mathrm{~min}$. Samples were then placed for $20 \mathrm{~min}$ in a $4^{\circ} \mathrm{C}$ block to aid protein precipitation and then centrifuged at $13,400 \times g$ for $10 \mathrm{~min}$ at $4^{\circ} \mathrm{C}$ to pellet the protein. The supernatant was taken and placed in a clean $2 \mathrm{ml}$ glass vial and was used for derivatization.

\subsection{Preparation of the methyl ester derivative and ${ }^{13} \mathrm{C}$ methyl ester of EDTA}

The methylation of EDTA was prepared by a simple derivatization procedure. Both the standard and isolated EDTA samples were dried at $60^{\circ} \mathrm{C}$ using a SpeedVac (Jouan RC1010) and reconstituted in $3 \mathrm{M}$ 
hydrochloric acid in methanol $(1 \mathrm{ml})$. The reaction mixture was incubated for 4 hours at $65^{\circ} \mathrm{C}$ in a capped $2 \mathrm{ml}$ glass vial followed by evaporation to dryness. The derivatized EDTA sample was reconstituted in water and a series of working solutions were prepared. The $\left[{ }^{13} \mathrm{C}\right]$-methyl ester analogue of EDTA was used as an internal standard and was prepared using $\left[{ }^{13} \mathrm{C}\right]$-methanol as the reagent for the derivatization procedure.

\subsection{Butylation of EDTA}

EDTA standard $(1000 \mu \mathrm{M})$ in water was dried and reconstituted in $1 \mathrm{ml}$ of $3 \mathrm{M}$ hydrochloric acid in butanol. Derivatization was performed in the same manner as methylation.

\subsection{DIOS surface preparation}

The DIOS chip preparation has been published earlier $[9,16]$. Briefly, photopatterning of the silicon wafer was achieved by etching low resistivity $(0.005-0.02 \Omega$-cm) n-type $\mathrm{Si}(100)$ wafers in $25 \% \mathrm{v} / \mathrm{v}$ hydrofluoric acid (HF)/ethanol through a 100 spot mask with white light illumination at a current density of $5 \mathrm{~mA} / \mathrm{cm}^{2}$ for $2 \mathrm{~min}$. The hydride terminated chip was then washed with methanol and dried with a stream of $\mathrm{N}_{2}$ before oxidation by a stream of ozone. Following the initial etching and oxidation, chips were then re-etched in a $5 \% \mathrm{v} / \mathrm{v} \mathrm{HF} / \mathrm{H}_{2} \mathrm{O}$ solution and oxidised. DIOS chips were chemically modified with pentafluorophenylpropyldimethylchlorosilane to generate silylated DIOS surface [15].

\subsection{GC analysis of methyl ester EDTA standards}

An Agilent Technologies $6890 \mathrm{~N}$ gas chromatograph was used for the analysis. The analysis was achieved with a methylsilicone (HP-MS5) column (30 m; $0.25 \mathrm{~mm}$ ID; $0.25 \mu \mathrm{M}$ coating). The injector temperature was set at $290^{\circ} \mathrm{C}$ and auxiliary temperature at $280^{\circ} \mathrm{C}$. The initial oven temperature was at $50^{\circ} \mathrm{C}$ for $4 \mathrm{~min}$ and then increased at $20^{\circ} \mathrm{C} / \mathrm{min}$ to $300^{\circ} \mathrm{C}$ and held at $300^{\circ} \mathrm{C}$ for $10 \mathrm{~min}$. A splitless injection was used, injecting $1 \mu \mathrm{l}$ and acquisition delay was set for 4 minutes. Helium with a flow rate of $0.8 \mathrm{ml} / \mathrm{min}$ was used as carrier gas. The selected ion monitoring (SIM) mode was also applied for low concentration detection. Fragment peaks at $m / z 174$ and 176, for methylated EDTA and ${ }^{13} \mathrm{C}$ methylated EDTA respectively were used to generate the calibration curve.

\subsection{Analysis of EDTA methyl ester by DIOS-MS}

The DIOS chips were attached directly to a modified MALDI target plate with scotch tape and $0.5 \mu 1$ aliquots of sample were deposited on the porous surface and allowed to dry in air before DIOS analysis. A Voyager DE-STR (Applied Biosystems) time-of-flight reflectron mass spectrometer with a pulsed nitrogen laser operated at $337 \mathrm{~nm}$ was used for the DIOS experiments. A delayed extraction period of 10-100 ns was used to minimize the energy spread of the ions for optimum resolution. Mass spectra were generated by averaging 100-150 individual laser shots into a single spectrum.

\subsection{LC-MS and LC-MS/MS analyses}

LC-MS analysis of underivatized and derivatized EDTA was performed on a Micromass Q-ToF micro system equipped with a Z-spray electrospray source and a lockmass sprayer. The source temperature was set to $110^{\circ} \mathrm{C}$ with a cone gas flow of $50 \mathrm{l} / \mathrm{h}$, a desolvation gas temperature of $365^{\circ} \mathrm{C}$ and a nebulization gas 
flow of $350 \mathrm{l} / \mathrm{h}$. The capillary voltage was set at $2.8 \mathrm{kV}$ and the cone voltage at $25 \mathrm{~V}$. The collision energy was set at $16 \mathrm{~V}$. LC separation was performed using a Waters 2795 HPLC separation module, together with a $\mathrm{C}_{18}$ column (Symmetry ${ }^{\circledR}$ Column, $2.1 \times 100 \mathrm{~mm}, 3.5 \mu \mathrm{m}$ particle size, Waters, Milford, MA). Mobile phase A consisting of water/formic acid [100/0.1 (v/v)], and B consisting of acetonitrile/formic acid $[100 / 0.1(\mathrm{v} / \mathrm{v})]$ were used for positive ion analysis. The sample $(5 \mu \mathrm{l})$ was applied to the column and eluted at a flow rate of $250 \mu \mathrm{l} / \mathrm{min}$ under gradient conditions $5 \% \mathrm{~B}$ to $95 \%$ for 32 minutes. MS and MS/MS data was collected for the mass range $100-1000 \mathrm{~m} / z$. The analysis of the resulting total ion chromatogram (TIC) was performed manually by generating extracted ion chromatograms (EICs) of the expected ion.

\subsection{Quantitative analysis}

GC-MS calibration curve was constructed by plotting the peak area ratio of the analyte to the internal standard versus the EDTA concentration. LC-MS calibration curve was obtained by plotting the peak intensity ratio of $m / z 174$ to 176 versus concentration. All calibration curves were fitted to a nonweighted linear regression. The precision of the method was measured as relative standard deviation (RSD), which was obtained by calculating the percentage ratio of the standard deviation to the mean of a minimum of three replicate measurements.

\section{Results and discussion}

Underivatized EDTA can be identified in preserved biological samples analysed by LC-MS at the concentrations present in commercially available EDTA stoppered tubes $(3-7 \mathrm{mM})[10,11]$. However, EDTA present in planted blood samples can be at significantly lower level and therefore there is a need for a more sensitive method of analysis.

In our initial LC-MS investigations, we determined that EDTA in its free acid form was not detected readily at low concentrations from EDTA spiked serum samples. To improve our detection, EDTA was derivatized to its methyl and butyl ester forms (Scheme 1). Although the concept of derivatizing each of the carboxylic acids is rather simple, derivatization must go to completion for this method to be viable for quantitation. Derivatization parameters such as incubation times, temperature and reagent were examined to determine optimal parameters. DIOS-MS was used as the mass analysis method for these optimisation experiments, due to its high sensitivity and rapid sample analysis capabilities $[8,15]$. Incubation times of $1,2,3,4,6$, and 18 hours, temperatures of 65 and $90^{\circ} \mathrm{C}$, and derivatization reagents to facilitate the formation of the methyl and butyl esters were investigated. From these parameters it was determined that complete methylation (tetramethyl ester) is achieved with 4 hour incubation at $65^{\circ} \mathrm{C}$ (Fig. 1). However, under the same conditions, butylation did not result in complete derivatization (Fig. 1) possibly due to steric effects. To maximise butylation, an extended period of incubations were explored, yet under extensive reactive conditions, butylation did not go to completion. Ultimately, because of reduced incubation time for complete esterification, methylation was chosen for EDTA derivatization.

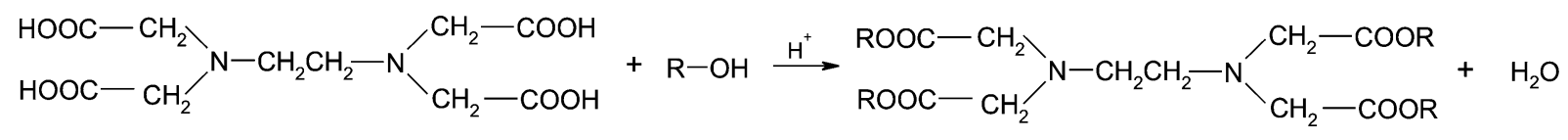

Scheme 1. Esterification reaction pathway of free acid EDTA, $\mathrm{R}=\mathrm{CH}_{3}$ or $\mathrm{CH}_{2} \mathrm{CH}_{2} \mathrm{CH}_{2} \mathrm{CH}_{3}$. 


\section{DIOS-MS}
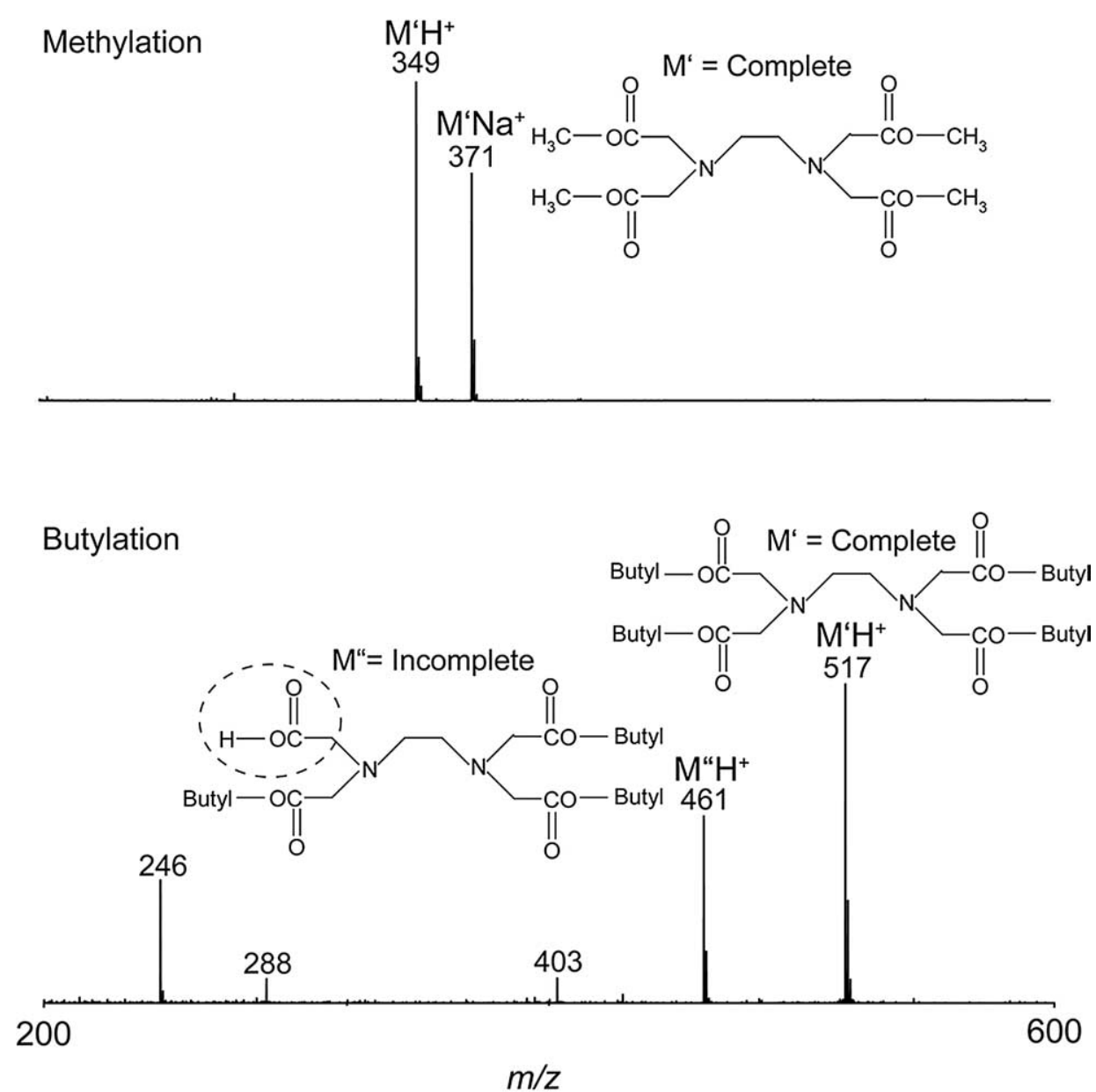

Figure 1. DIOS-MS of EDTA complete methylation at $m / z 349$ and DIOS-MS showing butylation not going to completion. Complete derivatization to the butyl ester $(\mathrm{m} / z$ 517) was not observed.

It should also be noted that for both esterification reactions, higher temperatures were less favourable because the EDTA ester became susceptible to water hydrolysis.

The simple methyl esterification reaction of EDTA allows for its analysis by GC-MS [14]. In addition, the derivatization procedure enables the inclusion of a stable isotope analogue as internal standard for quantitative analysis. Using $\left[{ }^{13} \mathrm{C}\right]$-labelled methanol, the $\left[{ }^{13} \mathrm{C}\right]$-EDTA internal standard can be readily synthesized. GC-MS was the method of analysis originally developed for the studies as it allows for the identification of molecules through retention time, formation of the molecular ion, and through the fragmentation pattern. The mass spectrum of the ester derivative of EDTA (Fig. 2) shows the precursor ion $\left(\mathbf{M}^{+}\right)$at $m / z 348$ and an intense fragment at $m / z 174$ which corresponds to the loss ${ }^{\circ} \mathrm{CH}_{2} \mathrm{~N}-$ $\left(\mathrm{CH}_{2} \mathrm{COOCH}_{3}\right)_{2}$. We also observed two prominent peaks at $m / z 289$ and 188 corresponding to the loss of a methyl ester and an $\mathrm{N}-\left(\mathrm{CH}_{2} \mathrm{COOCH}_{3}\right)_{2}$ group, respectively. Based on the scanning mode experiments on EDTA methyl ester standards, we obtained a detection limit (LOD) for the methyl ester 


\section{GC-MS}

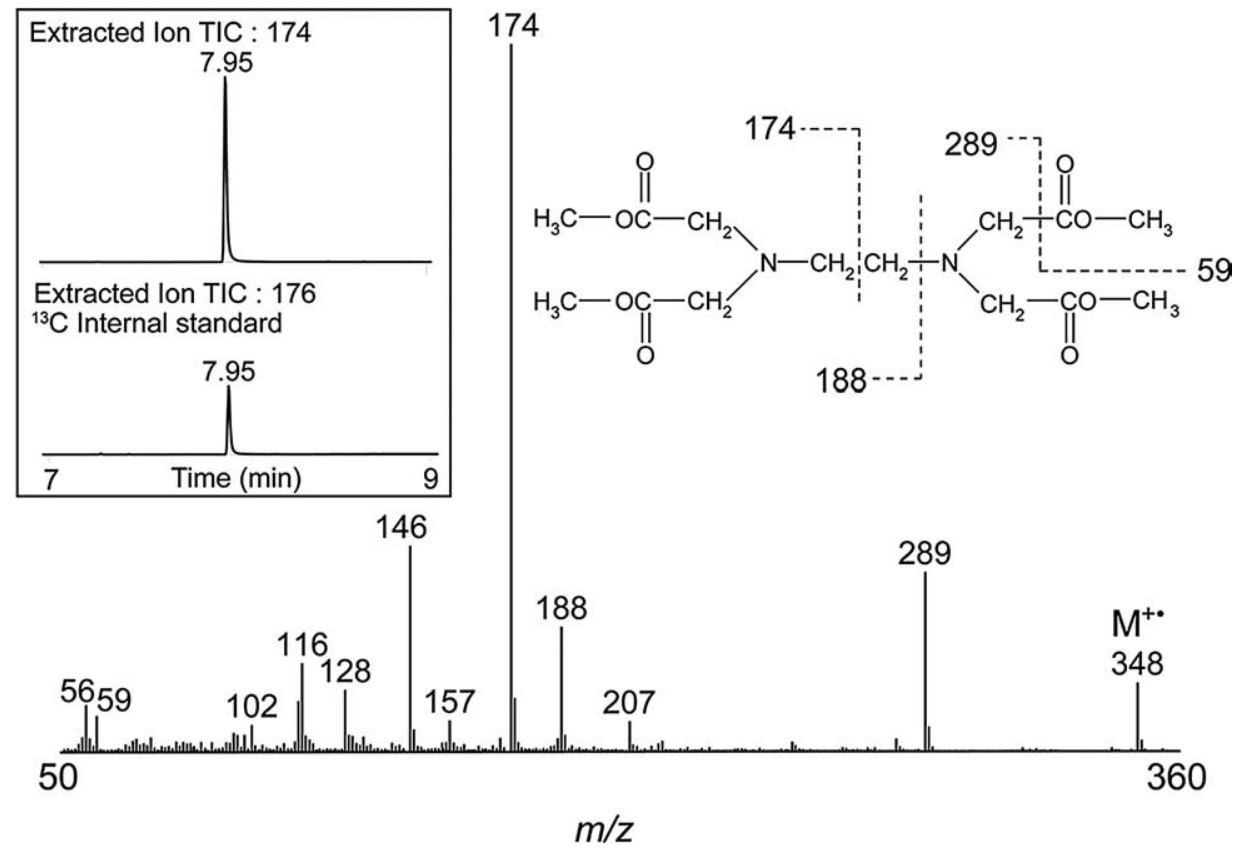

Figure 2. GC-MS fragmentation pattern of methylated EDTA. (Inset) SIM TIC of fragment ion methylated EDTA $(m / z$ 174) and $\left[{ }^{13} \mathrm{C}\right]$-analogue fragment ion $(\mathrm{m} / \mathrm{z} 176)$.

derivative of $1000 \mu \mathrm{M}$. To improve the analytical sensitivity, selective ion monitoring mode (SIM) was employed where a limit of detection of $0.5 \mu \mathrm{M}$ was achieved. The prominent peaks at $\mathrm{m} / z 174$ and 176 fragment ions of the methyl and $\left[{ }^{13} \mathrm{C}\right]$-methyl esters of EDTA, respectively, were then used for the quantitative analysis in serum. The SIM chromatograms in Fig. 2 (inset) depict a resolved baseline and the two co-eluting compounds of methyl ester derivative of EDTA and its $\left[{ }^{13} \mathrm{C}\right]$ analogue.

The optimized esterification method described above was used to generate the calibration curve for EDTA quantitative analysis in serum. Serum samples were spiked with EDTA at concentration of 0.5$100 \mu \mathrm{M}$ with a fixed amount of internal standard at $1.0 \mu \mathrm{M}$. The spiked serum samples were derivatized and analysed in triplicate. The calibration curve was constructed by plotting the peak area ratio of $m / z$ 174 and 176 versus the concentration. Figure 3a shows a linear response with a regression equation of $y=0.34575 x+0.31242$ and correlation coefficient of $R=0.99184$ in the working concentration range. The measured \% RSD at a given concentration shown in Table 1 is $20 \%$ or lower indicating good precision in the data. These results indicate the utility of the method in the detection of EDTA in complex matrices.

The analysis was also developed on the LC-MS/MS platform to broaden the applicability of the derivatization approach. Unlike GC-MS, EDTA in its free acid form can be detected using LC-MS, but only at concentrations above $100 \mu \mathrm{M}$. Figure 4a shows the LC-MS TIC of serum spiked with $10 \mu \mathrm{M}$ EDTA and $1 \mu \mathrm{M}\left[{ }^{13} \mathrm{C}\right]$-EDTA (internal standard) methyl ester derivatives. The mass spectrum of methylated EDTA is characterized by an intense protonated peak at $m / z 349$ and a sodiated peak at $m / z 371$. The protonated peak of the internal standard was observed at $m / z 353$ while the sodiated peak at $m / z 375$. The fragmentation profile of the methyl ester derivative of EDTA $(m / z 349)$ and of its $\left[{ }^{13} \mathrm{C}\right]$ analogue 
a. GC-MS

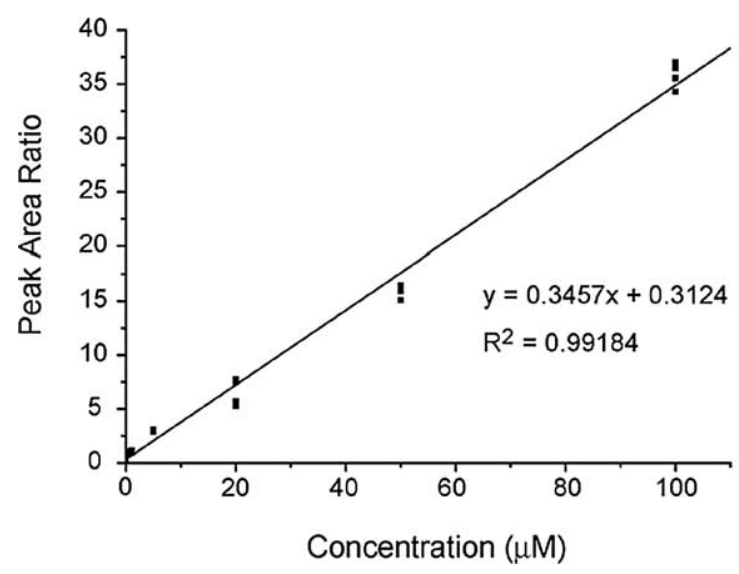

b. LC-MS

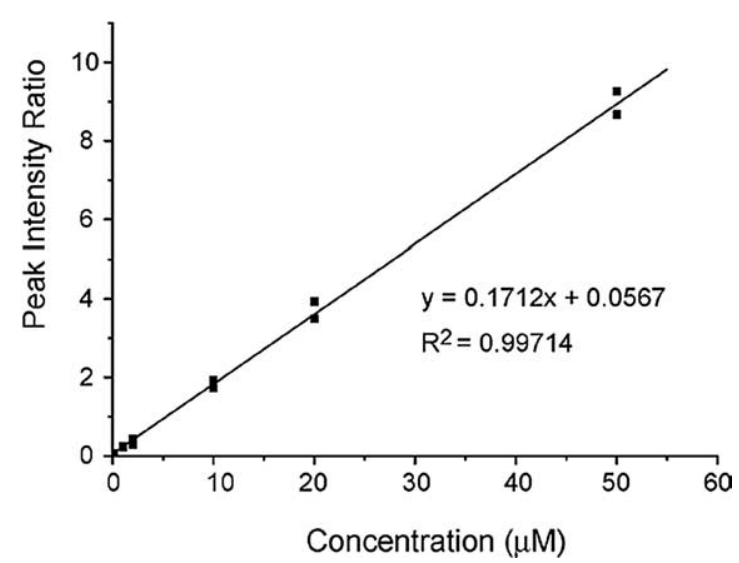

Figure 3. Plots of concentration vs. (a) peak area ratio using GC-MS and (b) peak intensity ratio using LC-MS of EDTA spiked serum samples.

Table 1

Sample-to-sample reproducibility in the quantitative analysis of EDTA in serum

\begin{tabular}{ccr}
\hline MS analysis & Concentration $(\mu \mathrm{M})$ & $\%$-RSD \\
\hline GC-MS & 0.5 & 14.5 \\
& 1.0 & 6.7 \\
& 5.0 & 3.3 \\
& 20.0 & 17.6 \\
LC-MS & 50.0 & 3.7 \\
& 100.0 & 5.5 \\
& 0.1 & 12.8 \\
& 1.0 & 5.5 \\
& 2.0 & 18.7 \\
& 10.0 & 6.3 \\
& 20.0 & 6.6 \\
& 50.0 & 3.9 \\
\hline
\end{tabular}

$(m / z$ 353) were generated using collision induced dissociation on an ESI-Q-ToF gave fragments at $m / z$ 188 and 190, respectively corresponds to the loss of $\mathrm{N}-\left(\mathrm{CH}_{2} \mathrm{COOCH}_{3}\right)_{2}$ (Fig. 4b).

A calibration curve of EDTA methyl ester in serum was generated showing an limit of quantitation (LOQ) of $0.1 \mu \mathrm{M}$. Serum samples were spiked with EDTA with concentration in the range of 0.01$50 \mu \mathrm{M}$ and a fixed amount of internal standard at $5 \mu \mathrm{M}$ were derivatized and analysed in four replicates. The calibration curve was constructed by plotting the MS/MS peak intensity of $m / z 174$ and $176 \mathrm{vs}$. the concentration ratio. Figure $3 \mathrm{~b}$ shows a linear response with a regression equation of $y=0.1712 x+$ 0.0567 and correlation coefficient of $R=0.99714$ in the working concentration range. The measured \% RSD at a given concentration shown in Table 1 is $20 \%$ or lower again indicating good precision in the data. 


\section{LC-MS}

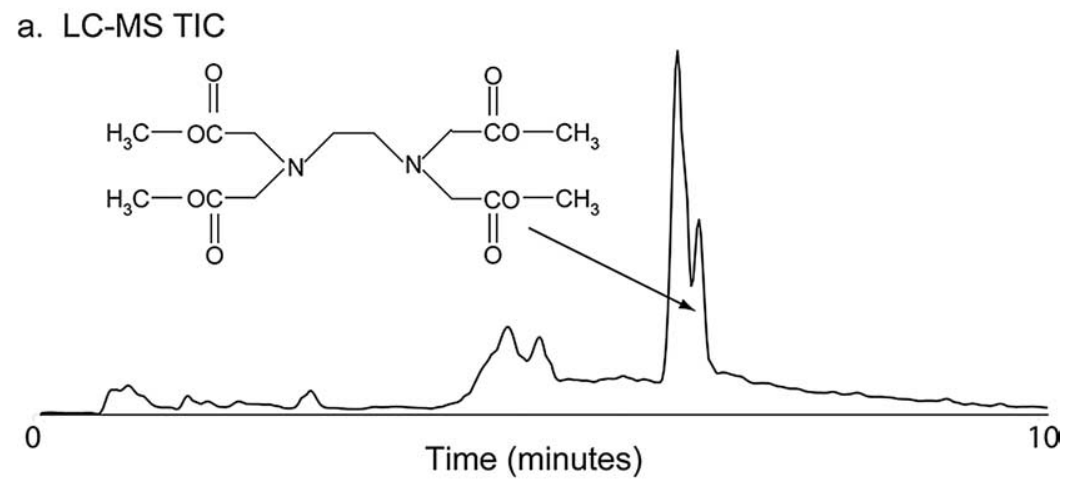

b. LC-MS spectra

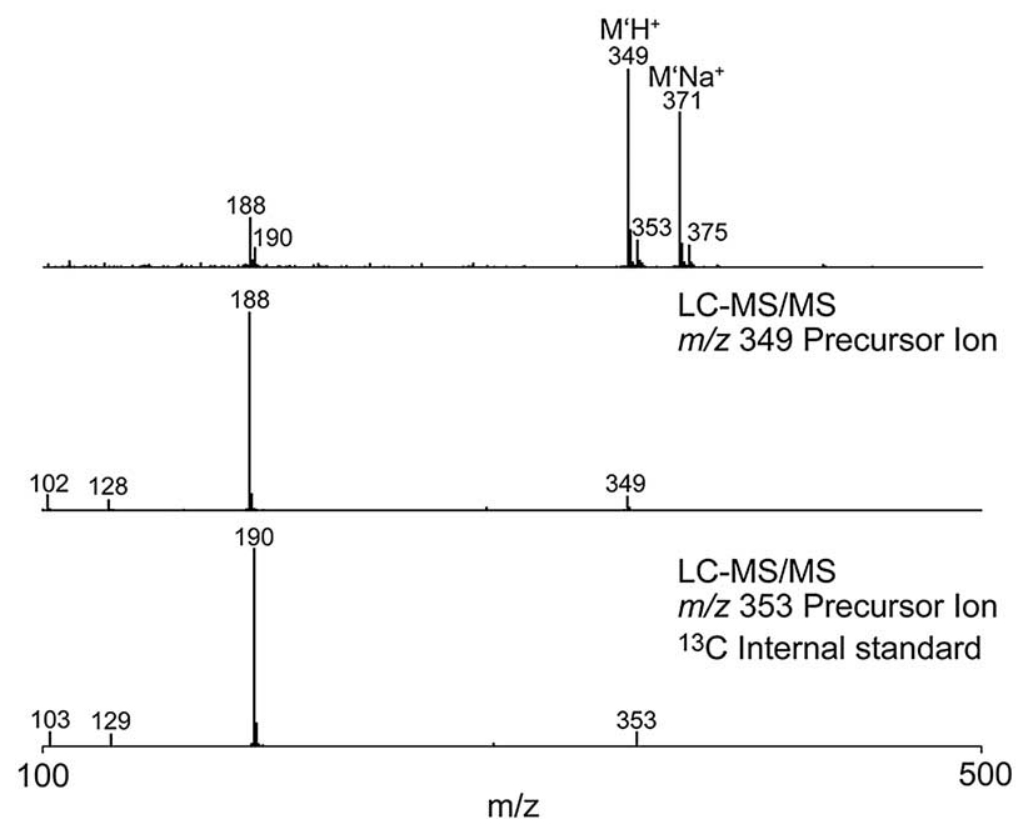

Figure 4. (a) LC-MS TIC of methylated EDTA spiked serum sample with $1 \mu \mathrm{M}\left[{ }^{13} \mathrm{C}\right]$-methylated analogue as internal standard. (b) LC-MS and tandem LC-MS mass spectra of the methylated and $\left[{ }^{13} \mathrm{C}\right]$-methylated EDTA protonated ions at $\mathrm{m} / z 349$ and 353 , respectively.

A standard part of criminal investigations involving serum analysis is both EDTA analysis as well as alternative testing methods to measure blood amount present. During the course of the LC-MS/MS and DIOS-MS studies it should be noted that we readily observed numerous endogenous metabolites in serum from the methanol extract (without derivatization). Among the most ubiquitous metabolites we observed two phospholipids - 1-palmitoyl-sn-glycero-3-phosphocholine at $m / z 496$ and 1-stearoyl-snglycero-3-phosphocholine at $m / z 524$ (Fig. 5). The MS/MS spectra of both metabolites and standards helped confirm the identification of both metabolites. 


\section{a. LC-MS}

1-Palmitoyl-sn-glycero-3-phosphocholine 1-Stearoyl-sn-glycero-3-phosphocholine
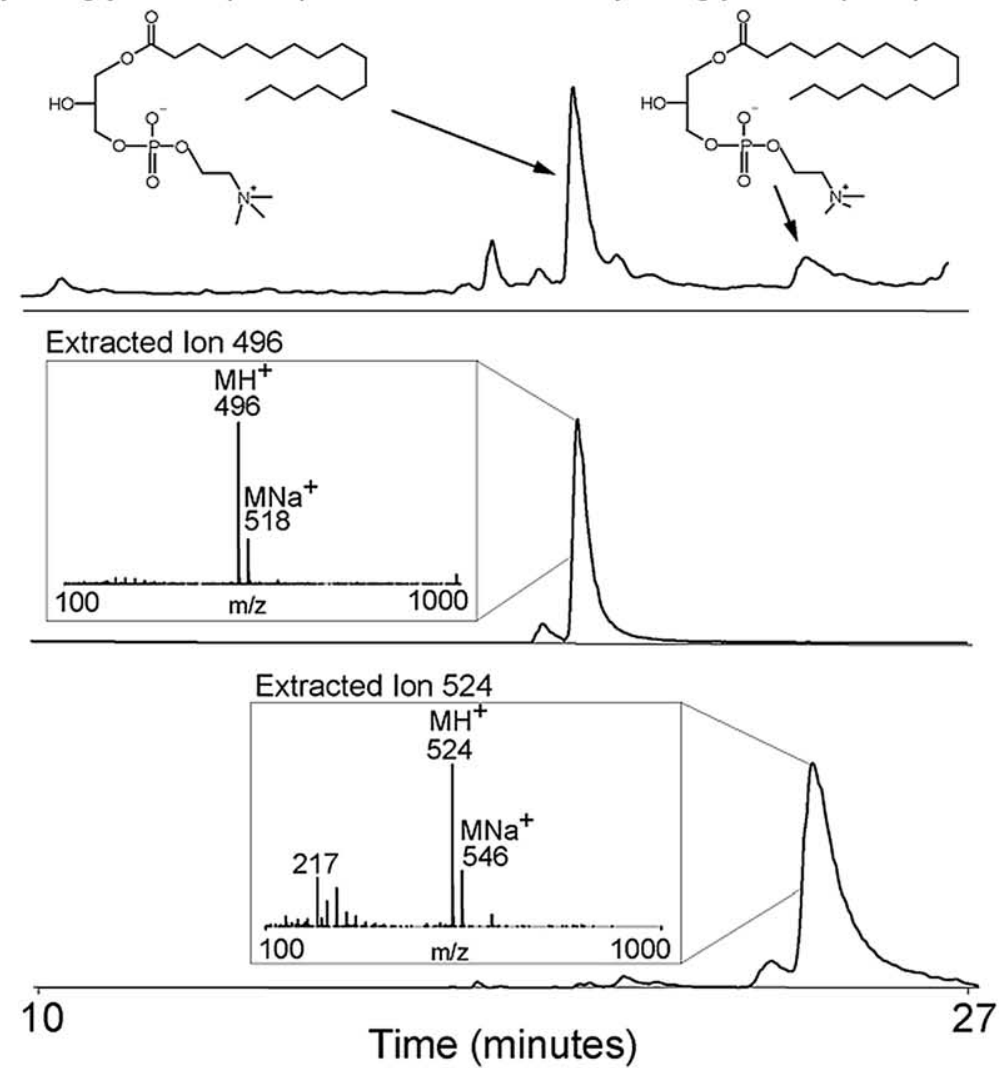

b. DIOS-MS

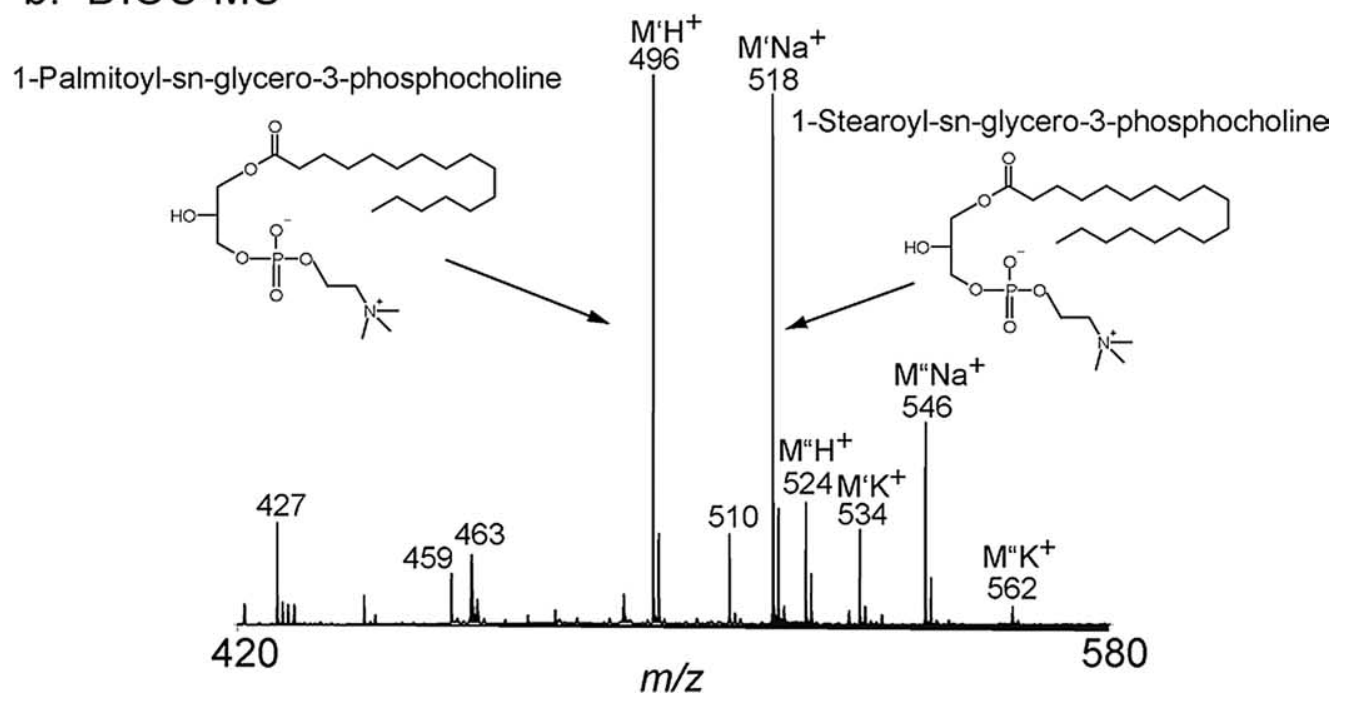

Figure 5. Metabolites identified in methanol extract, 1-palmitoyl-sn-glycero-3-phosphocholine $(\mathrm{m} / z$ 496) and 1-stearoyl-snglycero-3-phosphocholine ( $m / z$ 524) using (a) LC-MS and (b) DIOS-MS. 


\section{Conclusions}

This study demonstrates the feasibility for performing a simple methyl ester derivatization of EDTA from a complex mixture that enables enhanced detection from three different mass spectrometry platforms. Quantitative analysis was also readily performed for EDTA detection from the serum samples. The experiments also revealed the use of ubiquitous metabolites as potential biomarkers correlating with the presence of serum.

\section{Acknowledgement}

The authors appreciate partial funding for this research through NTH grant MH073490 and Mass Consortium Corporation.

\section{References}

[1] K.E. Grant, R.B. Lucke, S.A. Clauss, G.M. Mong, B.D. Lerner and J.A. Campbell, J. Radioanal. Nuc. Chem. - Articles 207 (1996), 247-261.

[2] L. Hall and L. Takahashi, J. Pharm. Sci. 77 (1988), 247-250.

[3] B.T. Hunter, Consumer's Res. 71 (1988), 8-9.

[4] A.S. Kord, I. Tumanova and W.L. Matier, J. Pharm. Biomed. Anal. 13 (1995), 575-580.

[5] H.L. Levy and S. Albers, Annu. Rev. Genom. Hum. Genet. 1 (2000), 139-177.

[6] M.L. Miller, B.R. McCord, R. Martz and B. Budowle, J. Anal. Toxicol. 21 (1997), 521-528.

[7] W.H. Seegers, Blood Clotting and Enzymology, Academic Press, New York, 1967, pp. 364-366.

[8] Z.X. Shen, E.P. Go, A. Gamez, J.V. Apon, V. Fokin, M. Greig, M. Ventura, J.E. Crowell, O. Blixt, J.C. Paulson, R.C. Stevens, M.G. Finn and G. Siuzdak, Chembiochem 5 (2004), 921-927.

[9] Z.X. Shen, J.J. Thomas, C. Averbuj, K.M. Broo, M. Engelhard, J.E. Crowell, M.G. Finn and G. Siuzdak, Anal. Chem. 73 (2001), 612-619.

[10] R.L. Sheppard and J. Henion, Anal. Chem. 69 (1997), 2901-2907.

[11] R.L. Sheppard and J. Henion, Anal. Chem. 69 (1997), A477-A480.

[12] R.L. Sheppard and J. Henion, Electrophoresis 18 (1997), 287-291.

[13] M. Sillanpaa, K.B. Raimo and M.L. Sihvonen, Anal. Chim. Acta 303 (1995), 187-192.

[14] J. Sorvari, M. Sillanpaa and M.L. Sihvonen, Analyst 121 (1996), 1335-1339.

[15] S.A. Trauger, E.P. Go, Z.X. Shen, J.V. Apon, B.J. Compton, E.S.P. Bouvier, M.G. Finn and G. Siuzdak, Anal. Chem. 76 (2004), 4484-4489.

[16] J. Wei, J.M. Buriak and G. Siuzdak, Nature 399 (1999), 243-246.

[17] The Versenes, Powerful Organic Chelating Agents for Extracting Control Cations, Framingham, Bersworth Chemical Company, MA, 1954. 


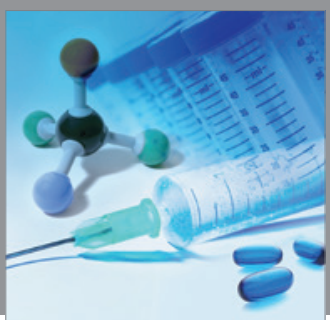

International Journal of

Medicinal Chemistry

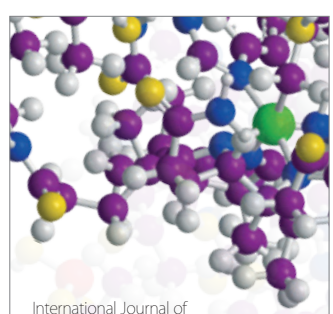

Carbohydrate Chemistry

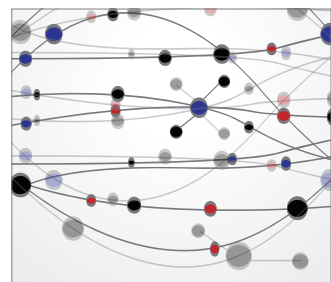

The Scientific World Journal
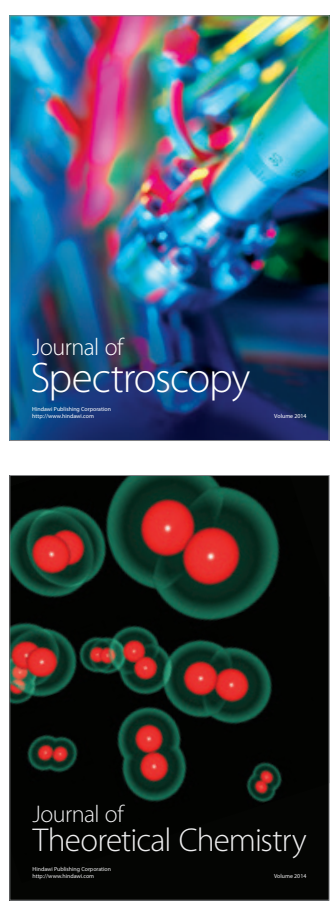
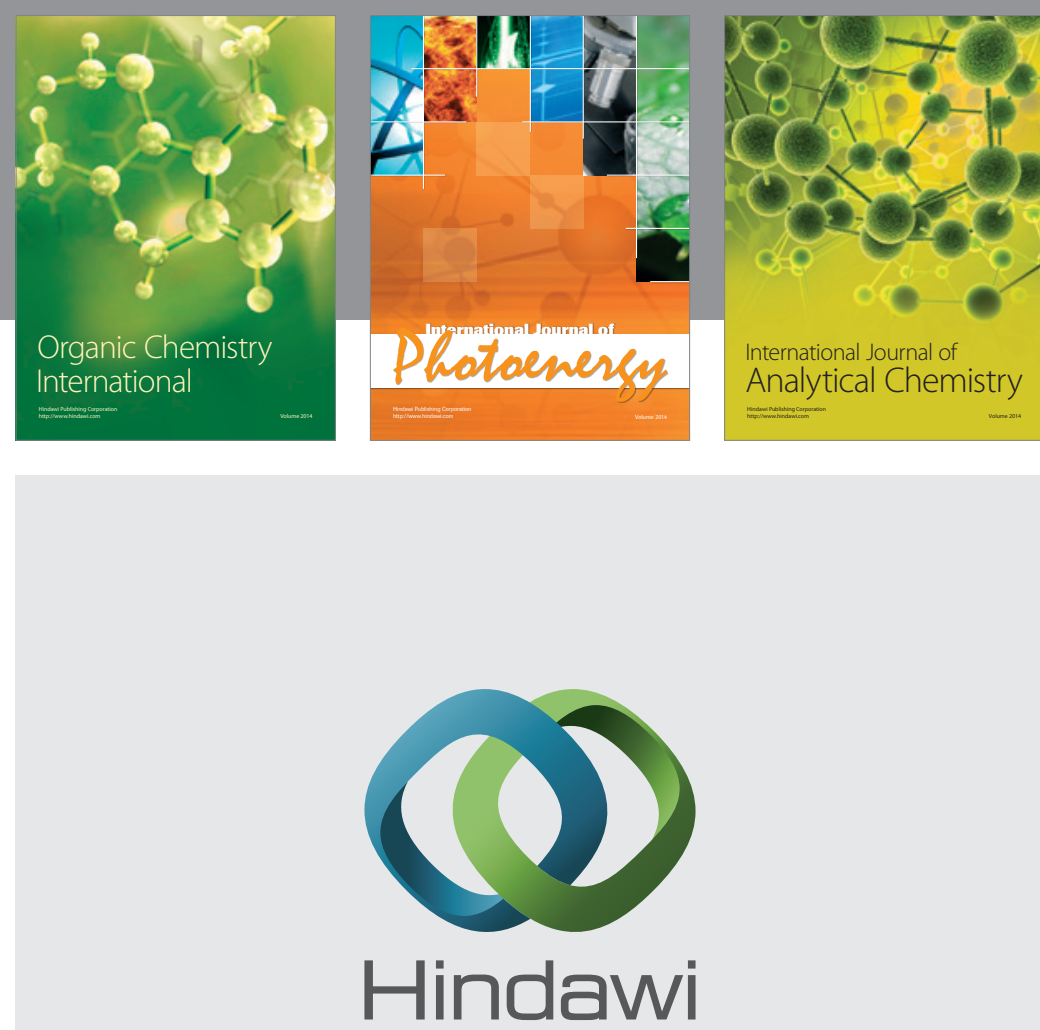

Submit your manuscripts at

http://www.hindawi.com
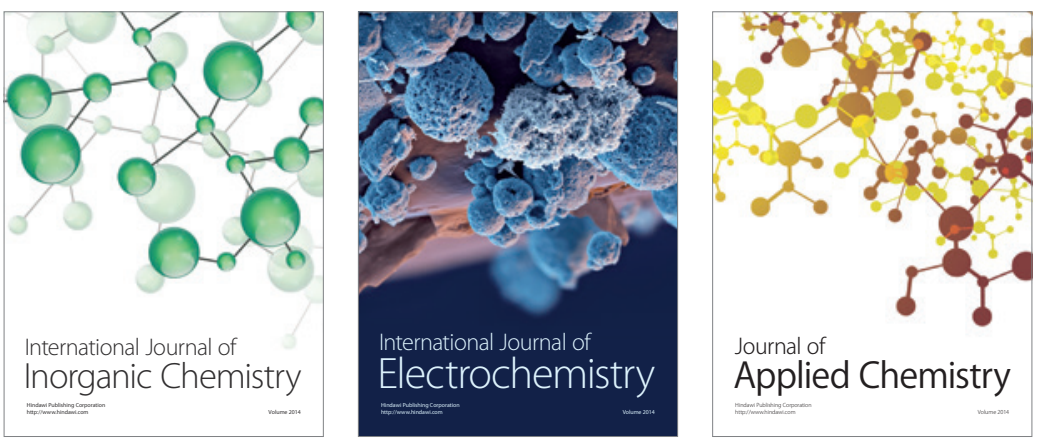

Journal of

Applied Chemistry
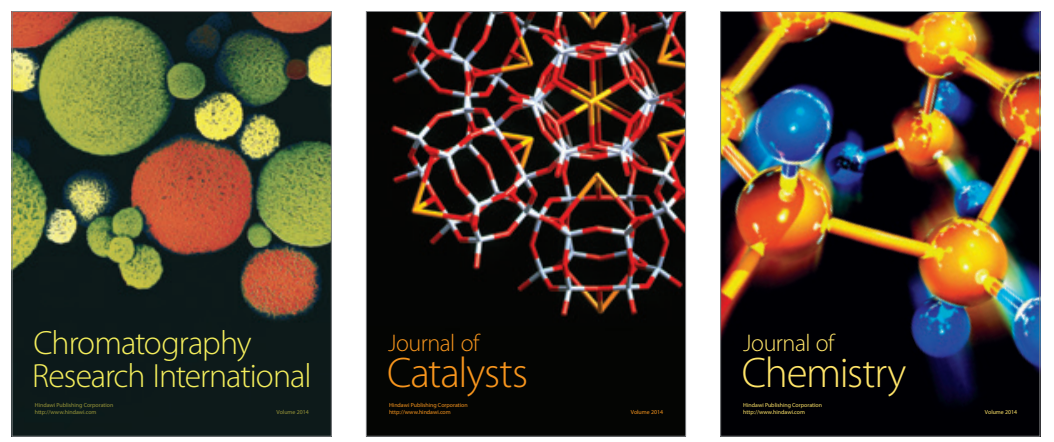
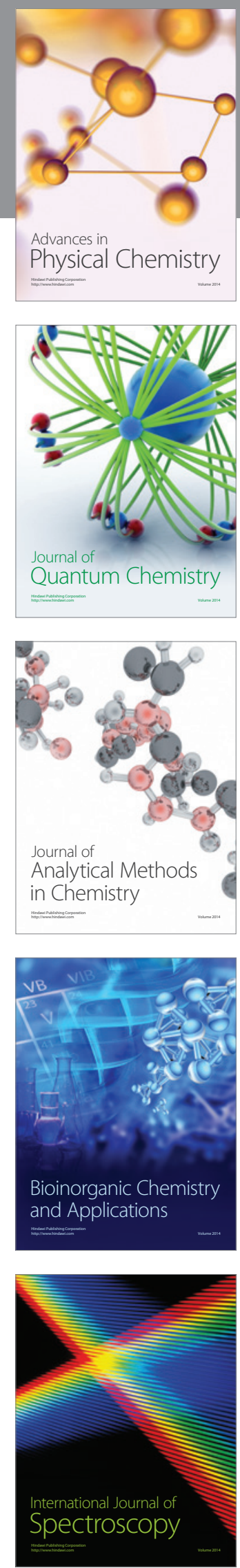